\title{
Dual roles of Sema6D in cardiac morphogenesis through region-specific association of its receptor, Plexin-A1, with off-track and vascular endothelial growth factor receptor type 2
}

\author{
Toshihiko Toyofuku, ${ }^{1,2,3,5}$ Hong Zhang, ${ }^{1,2,5}$ Atsushi Kumanogoh, ${ }^{2,3}$ Noriko Takegahara, ${ }^{2}$ \\ Fumikazu Suto, ${ }^{4}$ Junko Kamei, ${ }^{1,2}$ Kazuhiro Aoki, ${ }^{1}$ Masanori Yabuki, ${ }^{1,2}$ Masatsugu Hori, ${ }^{1}$ \\ Hajime Fujisawa, ${ }^{4}$ and Hitoshi Kikutani ${ }^{2,3,6}$ \\ ${ }^{1}$ Department of Internal Medicine and Therapeutics, Osaka University Graduate School of Medicine, 2-2 Yamada-oka, Suita, \\ Osaka 565-0871, Japan; ${ }^{2}$ Department of Molecular Immunology, Research Institute for Microbial Diseases, Osaka \\ University, 3-1 Yamada-oka, Suita, Osaka 565-0871, Japan; ${ }^{3}$ Core Research For Evolutional Science and Technology \\ (CREST), Japan Science and Technology Corporation, Japan; ${ }^{4}$ Division of Biological Science, Nagoya University Graduate \\ School of Science, Chikusa-ku, Nagoya 464-8602, Japan
}

Semaphorins, originally identified as axon guidance factors in the nervous system, play integral roles in organogenesis. Here, we demonstrate a critical involvement of Sema6D in cardiac morphogenesis. Ectopic expression of Sema6D or RNA interference against Sema6D induces expansion or narrowing of the ventricular chamber, respectively, during chick embryonic development. Sema6D also exerts region-specific activities on cardiac explants, a migration-promoting activity on outgrowing cells from the conotruncal segment, and a migration-inhibitory activity on those from the ventricle. Plexin-A1 mediates these activities as the major Sema6D-binding receptor. Plexin-A1 forms a receptor complex with vascular endothelial growth factor receptor type 2 in the conotruncal segment or with Off-track in the ventricle segment; these complexes are responsible for the effects of Sema6D on the respective regions. Thus, the differential association of Plexin-A1 with additional receptor components entitles Sema6D to exert distinct biological activities at adjacent regions. This is crucial for complex cardiac morphogenesis.

[Keywords: Semaphorin; Plexin; cardiac development]

Supplemental material is available at http://www.genesdev.org.

Received November 4, 2003; revised version accepted January 16, 2004.

The semaphorin family of proteins is characterized by a phylogenetically conserved sema domain in the extracellular region (Kolodkin et al. 1993). On the basis of additional structural features, such as the presence or absence of transmembrane domains, Ig-like domains, thrombospondin repeats, and glycophosphatidylinositol linkage sites, the family has been subdivided into eight groups, which also include virally derived proteins (Semaphorin Nomenclature Committee 1999). Despite the fact that a number of semaphorins have been known to play crucial roles in the hard wiring of the nervous systems, including fasciculation, axon branching, and target selection as axonal guidance cues (Pasterkamp and

\footnotetext{
${ }^{5}$ These authors contributed equally to this work.

${ }^{6}$ Corresponding author.

E-MAIL kikutani@ragtime.biken.osaka-u.ac.jp; FAX 81-06-6875-4465. Article published online ahead of print. Article and publication date are at http://www.genesdev.org/cgi/doi/10.1101/gad.1167304.
}

Kolodkin 2003), increasing evidence attested to the significance of semaphorins in the development and regulation of other organ systems. The Class III semaphorins, Sema3A, Sema3C, and Sema3F, are thought to regulate branching morphogenesis of the developing lung (Kagoshima and Ito 2001). Sema3A inhibits angiogenesis through competition for vascular endothelial growth factor (VEGF; Miao et al. 1999). Deletion of the Sema3C gene induces malformation of the cardiac outflow tract, suggesting Sema3C may control the migration of cardiac crest cells to this region (Behar et al. 1996). Sema3B and Sema3E are involved in tumor growth and metastasis (Christensen et al. 1998; Tse et al. 2002). Two class IV semaphorins, Sema4A and Sema4D, regulate aspects of the immune response (Kumanogoh et al. 2000; Shi et al. 2000). Sema4D also plays a role in the invasive growth of epithelial cells (Giordano et al. 2002). Although a wide range of biological functions are apparent, the molecular 
mechanisms governing the phase-specific and regionspecific operation of these signals, particularly those functioning in organogenesis outside of the nervous system, remain poorly characterized.

One of the approaches to the understanding of the molecular mechanisms governing the biological roles of semaphorins revolved around the profiling of the associated receptors. Neuropilin-1 (NP1) and neuropilin-2 (NP2) have been identified as high-affinity binding partners for class 3 semaphorins (Pasterkamp and Kolodkin 2003). A second class of semaphorin-binding proteins, the Plexins, has been identified as the primary receptors for semaphorins that do not bind NPs /Comeau et al. 1998). Fly Plexin-A is a receptor for Semala (Winberg et al. 1998), Plexin-B1 binds to Sema4D, and Plexin-C1 (virus-encoded semaphorin receptor) functions as a binding partner for Sema7A, as well as the viral semaphorins, SEMAVA (semaphorin A39R) and SEMAVB (AHV sema; Comeau et al. 1998). In the immune system, Sema4A and Sema4D use Tim2 and CD72, respectively, as receptors; neither of these receptors has any structural relationship to the neuropilin or Plexin families (Kumanogoh et al. 2000, 2002). Recent studies indicate that semaphorins signal through multimolecular receptor complexes. For example, NP1 requires Plexin-A1 to transduce Sema3A signals (Takahashi et al. 1999). L1, a member of the Ig superfamily of cell-adhesion molecules, associates with NP1 to modulate Sema3A signaling (Castellani et al. 2002). Drosophila Plexin-A forms a receptor complex with the receptor tyrosine kinase-like transmembrane protein Off-track (OTK) (Winberg et al. 2001). A receptor tyrosine kinase, c-Met, also associates with Plexin-B1 to function as a modulator of Sema4D signals mediating the invasive growth of epithelial cells (Giordano et al. 2002).

In this study, we isolated a class VI semaphorin, Sema6D, from the mouse heart, and assessed the role of
Sema6D in organogenesis, utilizing the whole chick-embryo culture system. Ectopic expression of Sema6D, as well as RNA interference (RNAi) against Sema6D, induced malformations in the cardiac tube. Furthermore, Sema6D was found to participate in cardiac morphogenesis by exerting distinct biological activities through its receptor, Plexin-A1, which formed receptor complexes with OTK and vascular endothelial growth factor receptor type 2 (VEGFR2) in adjacent regions of the cardiac tube.

\section{Results}

\section{Expression of Sema6D in mouse and chick embryos}

In search of semaphorins involved in cardiac development, we cloned the cDNA encoding the recently identified Sema6D (Qu et al. 2002) by RT-PCR of mouse heart mRNA and subsequent cDNA library screening. The human and chicken orthologs (hSema6D and cSema6D) were also isolated, exhibiting high amino acid sequence conservation $(99 \%$ and $98 \%$ homology to the mouse Sema6D, respectively).

The expression of Sema6D was first detected in the cardiac crescent and neural fold of embryonic day 9 (E9) mouse embryo (data not shown) and then became prominent in the neural fold, the atrial and ventricular vesicles, and the forelimb at E10.5 (Fig. 1A). Cross-sectioning indicated the expression of Sema6D in the dorsal side of neural fold (Fig. 1B-D). Sema6D mRNA was observed throughout the entire heart, including the conotruncal (CT) segment (Fig. 1C, arrow), the atrioventricular segment (Fig. 1D, arrow), and the ventricular myocardium (Fig. 1C,D, arrowheads). The expression of Sema6D was higher in myocardial cells (Fig. 1E, arrowhead) than in endocardial cells (Fig. 1E, arrow). cSema6D expression was detected at high levels in the dorsal side of the neu-

Figure 1. Sema6D mRNA is expressed in the developing neural and cardiac tubes. $(A)$ Lateral view of E10.5 mouse embryo. $(B-E)$ Cross-sections through the E10.5 mouse embryos shown in $A$. Sema6D mRNA is expressed in the dorsal side of the neural tube $(B-D)$ and the cardiac tube, including the CT segment $(C$, arrow), atrioventricular segment $(D$, arrow $)$, and ventricle $(C, D$, arrowheads). The magnified view of the ventricle $(E)$ demonstrates Sema6D mRNA expression in the myocardial cells in compact zone and trabeculae (arrowhead), more than in the endocardial cells (arrow). Bars: $A-D, 100 \mu \mathrm{m} ; E, 20$ $\mu \mathrm{m} .(F-H)$ Expression of cSema6D mRNA in $\mathrm{HH}$ stage $7(F, G)$ and $\mathrm{HH}$ stage $12(H)$ chicken embryos. Ventral view $(F)$ and cross-sections $(G, H)$ display cSema6D mRNA expression in the dorsal side of the neural fold $(G$, arrow) and the cardiac ventricular wall $(H$, arrow). The expression pattern of cSema6D mRNA in the chick embryo is similar to that seen in the mouse embryo. Bar, $100 \mu \mathrm{m}$.
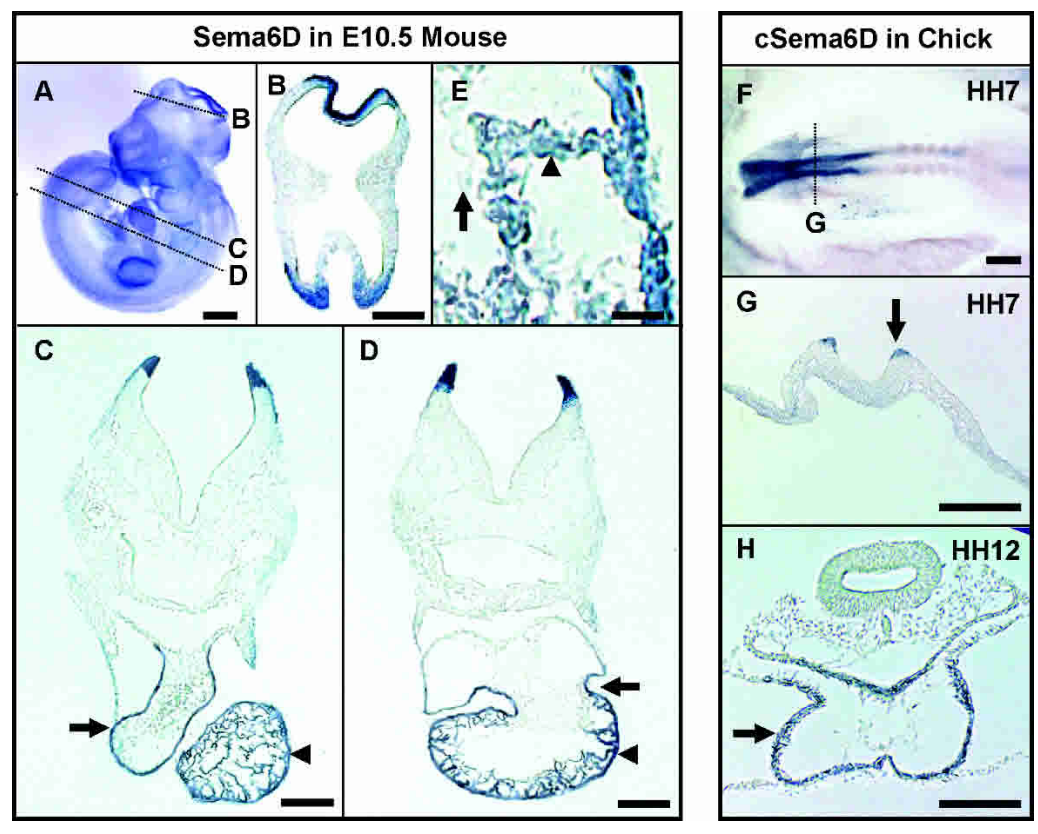
ral fold of Hamburger and Hamilton $(\mathrm{HH})$ stage 7 chick embryo (Fig. 1F, arrow in $1 \mathrm{G}$ ), and the cardiac tube wall of $\mathrm{HH}$ stage 12 embryo (Fig. 1H, arrow). Thus, Sema6D exhibited similar expression patterns in the developing embryos of chickens and mice.

\section{Ectopic Sema6D expression induces ventricular expansion and neural tube closure defect, whereas RNAi against Sema6D results in narrowing of ventricular cavity}

To examine the biological activities of Sema6D in embryonic development, chick embryos at $\mathrm{HH}$ stage 4 were implanted with cells expressing Sema6D or control cells and allowed to grow to $\mathrm{HH}$ stage 12 (Fig. 2A-F) or to $\mathrm{HH}$ stage 16 (Fig. 2G-I). In vertebrate embryos, the cardiac mesoderm first positions bilaterally in the anterior lateral plate mesoderm ( $\mathrm{HH}$ stage 4 of the chick embryo), then migrates toward the midline to form the definitive cardiac tube (HH stage 10; Schultheiss and Lassar 1999). Subsequently, the heart tube begins to generate a rightside bend at $\mathrm{HH}$ stage 12 , nearly completing the looping at $\mathrm{HH}$ stage 16 as observed in normal embryos (data not shown) and control-cell implanted embryos (Fig. 2A,G). Presumptive cardiac regions position segmentally throughout the cardiac tube, beginning with the CT seg-
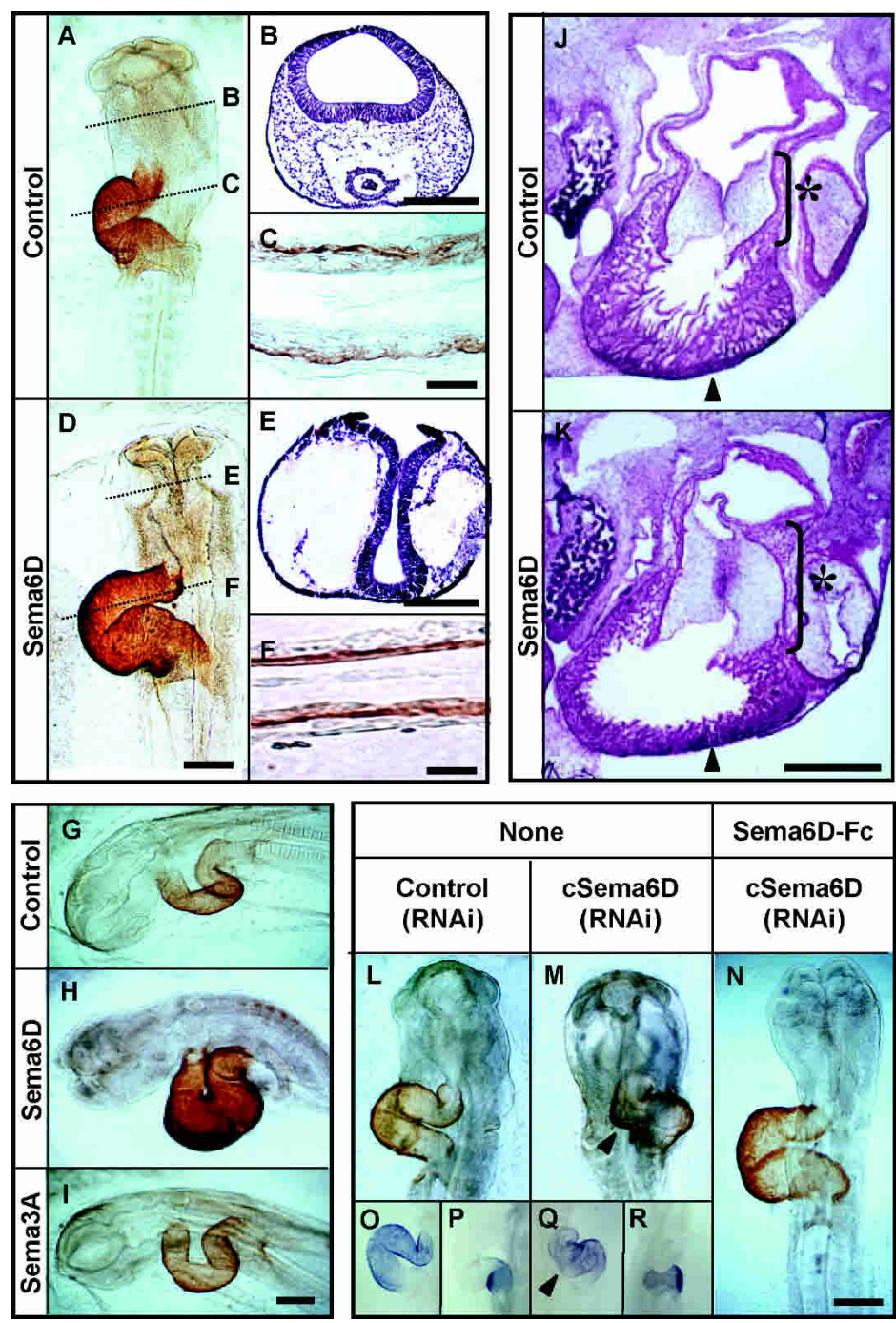
with either control or $(M, Q, R)$ or presen $\mathrm{HH}$ stage 12. Embryos were then immunostained with anti-vMHC antibody $(L-N)$ or in situ hybridized with riboprobes for VMHC1 $(O, Q)$ or AMHC1 $(P, R)$. Ventral view of embryos treated with siRNA specific for cSema6D display a narrowing of the ventricle $(M$, arrowhead) in comparison with the right-side bended cardiac tube in embryos treated with a control siRNA $(L)$. Note that siRNA specific for cSema6D distorted exclusively the ventricular portion $(Q$, arrowhead) but not the atrial portion $(R)$, compared with control siRNA $(O, P)$. In the presence of Sema6D-Fc, embryos treated with siRNA specific for cSema6D display nearly normal or expanded cardiac tube $(N)$. Bar, $100 \mu \mathrm{m}$.

Figure 2. Ectopic expression of Sema6D and RNAi against cSema6D result in abnormal cardiac tube formation. $(A-F) \mathrm{HH}$ stage 12 chick embryos implanted with control cells $(A-C)$ or Sema6D-expressing cells $(D-F)$. HH stage 4 embryos were removed from eggs, placed in the culture dishes, and implanted with cells $\left(10^{6}\right.$ cells/aggregate) on the edge of the lateral mesoderm. Embryos were cultured until $\mathrm{HH}$ stage 12 and either immunostained with anti-vMHC antibody $(A, C, D, F)$ or visualized with hemotoxylene and eosin staining $(B, E)$. Cross-sections through each embryo are indicated in the respective ventral view. Ventral view $(D)$ and cross-section $(E)$ of embryos implanted with Sema6D-expressing cells display defects in the neural tube closure and expansion of the cardiac cavity in comparison with embryos implanted with control cells $(A, B)$. Magnified view of ventricular wall of Sema6D-treated embryos $(F)$ also exhibit thin myocardial layer. Bars: $A, B, D, E, 100 \mu \mathrm{m} ; C, F, 20 \mu \mathrm{m}$. (GI) HH stage 16 chick embryos implanted with control $(G)$, Sema6D-expressing $(H)$, or Sema3A-expressing cells $(I)$. $\mathrm{HH}$ stage 4 embryos were treated as described above. Embryos were cultured until $\mathrm{HH}$ stage 16 and then immunostained with anti-vMHC antibody. Embryos implanted with Sema6D-expressing cells $(H)$ display a phenotype similar to that of $\mathrm{HH}$ stage $12(D)$, whereas embryos implanted with Sema3A-expressing cells $(I)$ display no apparent changes in either neural or cardiac morphology, in comparison with control embryos $(G)$. Bar, $100 \mu \mathrm{m} .(J, K)$ $\mathrm{HH}$ stage 36 chick embryos treated with control $(J)$ or Sema6D-expressing cells $(K)$. Sema6D-expessing cells or control cells $\left(10^{6}\right.$ cells $)$ were injected into the space between the vitelline membrane and chick embryo at $\mathrm{HH}$ stage 12. Embryos were allowed to develop in ovo up to $\mathrm{HH}$ stage 36. Cross-sections of embryos were visualized by hematoxylene and eosin staining. In Sema6D-treated embryos, cardiac ventricle exhibits expanded cavity and poor myocardial trabeculae $(K$, arrowhead) and the endocardial cushion of the CT segment is extended $(K$, asterisk), compared with the respective regions in control embryo $(J)$. Bar, $100 \mu \mathrm{m}$. $(L-R) \mathrm{HH}$ stage 12 chick embryos treated with control siRNA $(L, O, P)$ or siRNA specific for 
ment, through the primitive ventricle, atrioventricular canal, atrium, and sinus venosus. However, ectopic expression of Sema6D resulted in the expansion of the cardiac tube, particularly the primitive ventricle (Fig. 2D, $\mathrm{H}_{\text {; }}$ Supplementary Table 1), characterized by a thinner myocardial layer (Fig. 2F) than that of control embryos (Fig. 2C). In addition, failure of neural tube closure was observed (Fig. 2E). Incubation with soluble Sema6D-Fc (Fig. 4C, panel b) or implantation with cSema6D-expressing cells gave a similar phenotype (data not shown). As Sema6D-expressing cells spontaneously released the soluble semaphorin ectodomain into the medium (Supplementary Fig. 1), the effect of implanted Sema6Dexpressing cells may be mediated largely by soluble Sema6D.

To study the effect of ectopic Sema6D expression on the later phases of cardiac development, when active trabeculation of the ventricular wall occurs (Fig. 2J,K), aggregates of either Sema6D-expressing cells or control cells were injected into the amniotic cavity at $\mathrm{HH}$ stage 12. The embryos were then allowed to develop in ovo up to $\mathrm{HH}$ stage 36 . In control embryos of $\mathrm{HH}$ stage 36 , the primitive ventricular chambers exhibited trabeculation (Fig. 2J, arrowhead). In addition, the CT segment and atrioventricular canal fuse to generate the endocardial cushion, the primordial valve, and septa (Fig. 2J, asterisk; Sedmera et al. 2000). Embryos injected with Sema6Dexpressing cells displayed a larger ventricular chamber size with a decreased density of trabeculae (Fig. $2 \mathrm{~K}$, arrowhead). The endocardial cushion of Sema6D-treated embryos was also substantially extended, although an apparent change in its thickness was not observed (Fig. $2 \mathrm{~K}$, asterisk) compared with that of control embryos (Fig. 2J, asterisk). Thus, overexpression of Sema6D affects not only the ventricular region, but also the endocardial cushion region.

To further analyze the involvement of endogenous Sema6D in cardiac development, we next utilized RNAi to down-regulate cSema6D expression by introducing small interfering RNAs (siRNA) specific for cSema6D into the primitive cardiac tube by electroporation at $\mathrm{HH}$ stage 7 . This method delivers siRNA efficiently into cardiac tubes (Supplementary Fig. 2A). siRNA specific for cSema6D significantly reduced cSema6D mRNA levels without changing the expression of other mRNAs (Supplementary Fig. 2B). In embryos treated with a control siRNA oligonucleotide whose sequence was not complimentary to any host mRNAs, the cardiac tube developed normally (Fig. 2L). RNAi against cSema6D, however, resulted in distorted bending of cardiac tubes (Fig. 2M, arrowhead). To determine which segment of the cardiac tube is affected by RNAi, some of the embryos were analyzed by in situ hybridization using segment-specific riboprobes, ventricle myosin heavy chain-1 (VMHC1), and atrial myosin heavy chain-1 (AMHC1). Compared with the normal cardiac tube (Fig. $2 \mathrm{O}, \mathrm{P})$, the cardiac tube treated with siRNA specific for cSema6D was characterized by the small distorted ventricular portion connected with the normal caudal portion containing atria and sinus venosus of the cardiac tube (Fig. 2Q, [arrowhead], R; Supplementary Table 1). It is noteworthy that incubation with soluble Sema6D could correct or, rather, overcome the abnormality in cardiac tubes electroporated with siRNA for cSema6D (Fig. $2 \mathrm{~N})$, yet, in turn, defected neural tube closure as seen in embryos with overexpression of Sema6D. These results indicate that Sema6D is required for normal cardiac development.

\section{Sema6D displays distinct effects on explants from different regions of the cardiac tube}

Cardiac abnormalities induced by overexpression of Sema6D or RNAi against cSema6D might result from the failure of the coordinated differentiation of each cardiac segment. We then examined the effect of Sema6D on explants from the CT segment and ventricle of the cardiac tube. Explants were placed adjacent to control or Sema6D-expressing cells in collagen gel cultures (Fig. 3). We observed the outgrowth of epithelioid cells around the cardiac explants from both regions when cultured alone (Fig. 3A, panels a,e) or adjacent to control cells for $48 \mathrm{~h}$ of culture (Fig. 3A, panels b,f). When cultured with Sema6D-expressing cells, explants from the CT segment demonstrated enhanced outgrowth (Fig. 3A, panels c,i). This stimulatory effect of Sema6D was also observed on the outgrowth from explants of the atrioventricular (AV) canal, a cardiac cushion-forming region other than CT segment (Supplementary Fig. 3). In contrast, Sema6Dexpressing cells inhibited outgrowth from ventricle explants (Fig. 3A, panels g,i). Sema3A-expressing cells did not affect explant outgrowth (Fig. 3A, panels d,h,i).

The effects of Sema6D on outgrowing cells appear to reflect changes in cell migration rather than in cell proliferation, because Sema6D did not significantly affect the number of histone H3-positive proliferating cells in the outgrowth (Fig. 3A, panel j). To determine the cell type outgrowing in response to these cocultures, we performed immunohistochemistry of cardiac explants with antibodies specific for factorVIII (an endothelial cell marker), ventricular myosin heavy chain (vMHC; a cardiac myocyte marker), and fibrillin-2 (a mesenchymal cell marker; Fig. 3B). The outgrowing cells appeared to be endothelial rather than myocardial in nature, as they were stained with anti-factorVIII antibodies (Fig. 3B, panels a,c,e,g), but not anti-vMHC antibodies (data not shown). A population of fibrillin-2-positive mesenchymal cells were also identified in the migrating cells from the CT segment (Fig. 3B, panels b,f) and the ventricle (Fig. 3B, panels d,h). This indicates that Sema6D affects the migration of both endothelial and mesenchymal cells from the CT segment and ventricle explants. This effect of Sema6D was in contrast to that of TGF- $\beta$, which has been found to increase the mesenchymal cells from the CT segment (Brown et al. 1999); TGF- $\beta$ did not show a significant effect on the migration of endothelial cells from both explants (Supplementary Fig. 3). Thus, Sema6D differentially affects the CT segment and ventricular explants, exerting stimulatory and inhibitory ac- 

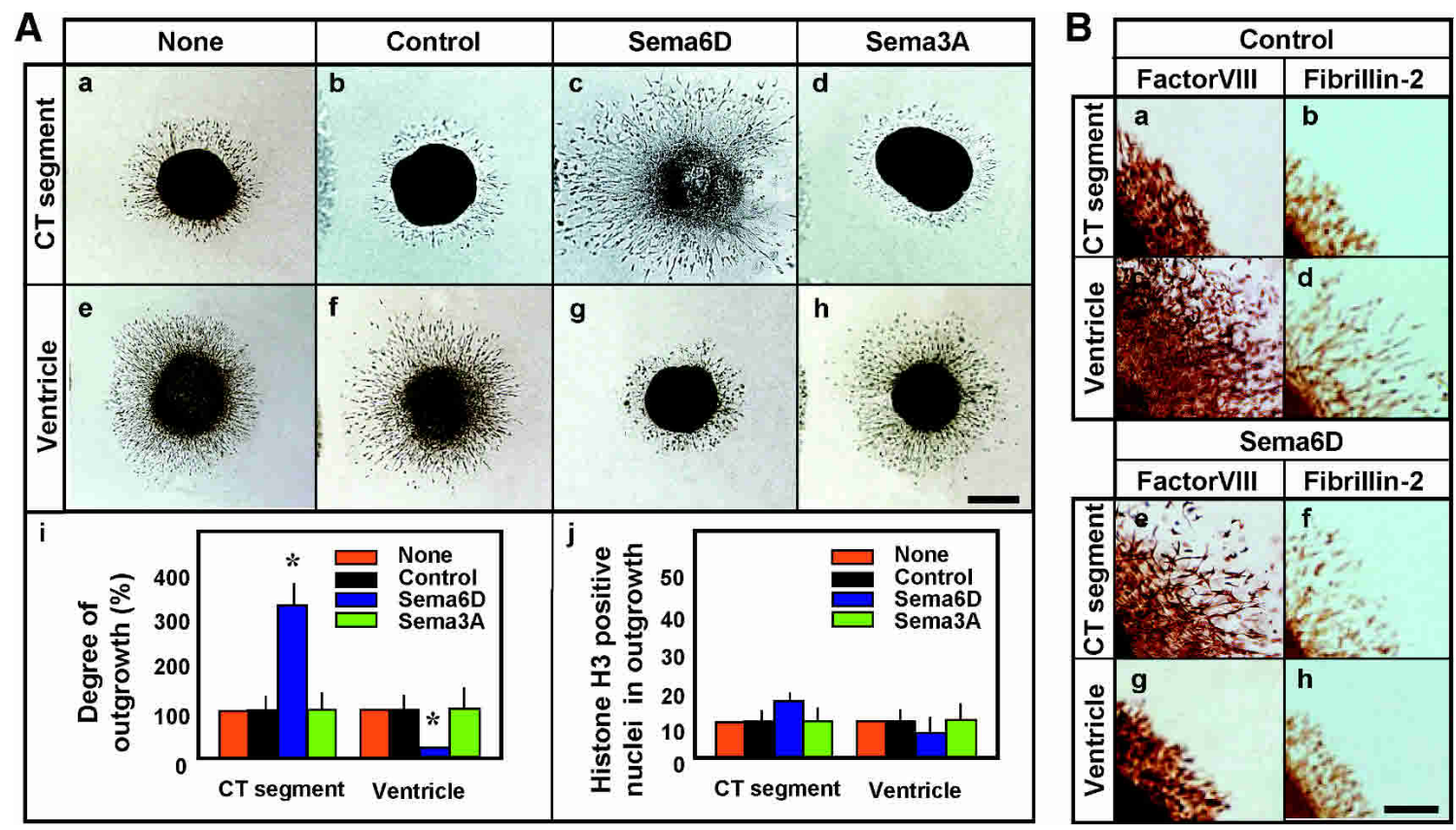

Figure 3. Sema6D induces distinct effects on the outgrowth from explants of different regions of cardiac tube. $(A$, panels $a-h)$ Collagen gel coculture of explants with control (panels $b, f$ ), Sema6D-expressing (panels $c, g$ ), or Sema3A-expressing cells (panels $d, h)$. Tissues were removed from chick embryos, placed on collagen gels, and cultured with medium. Explants were positioned on the right, whereas the cell aggregates (transfect.) were positioned on the left. Cardiac explants of the CT segment (panels $a-d$ ) and ventricle (panels $e-h$ ) were visualized by phase contrast microscopy. CT segment explants (panel $c$ ) demonstrate increased outgrowth toward the Sema6Dexpressing cells, whereas ventricular explants (panel $g$ ) exhibit decreased outgrowth. Bar, $100 \mu \mathrm{m}$. (Panels $i, j$ ) Quantification of the relative degree of outgrowth as a percentage of each control value (panel i) and total numbers of phospho-histone H3-positive nuclei within outgrowth (panel $j)$ of each explant. Data are means \pm S.E.M. $\left(^{\star}\right) p<0.05$, vs. explants with control cells. $(B$, panels $a-h)$ Immunohistochemistry of cardiac explant outgrowths. Cardiac explants were immunostained for factorVIII (panels $a, c, e, g$ ) or fibrillin-2 (panels $b, d, f, h$ ). CT segment explants exhibit increased numbers of factorVIII-positive cells (panel $e$ ), whereas ventricle explants demonstrate decreased numbers of factorVIII-positive cells in response to Sema6D (panel $\mathrm{g}$ ). Bar, $50 \mu \mathrm{m}$.

tivities on the migration of outgrowing cells from the former and latter, respectively.

\section{Sema6D interacts with Plexin-A expressed in the cardiac tube}

Members of the Plexin family serve as receptors or components of receptor complexes for semaphorins /Comeau et al. 1998). By RT-PCR of mouse heart mRNA using degenerative primers, we detected Plexin-A1 most frequently (51 of 96 tested clones), although Plexin-A2 $(n=15)$, Plexin-A4 $(n=18)$, and Plexin-B1 $(n=12)$ were also identified. We next examined the binding of Sema6D to the isolated Plexins. HEK293 cells transiently transfected with Plexin-A1, Plexin-A2, PlexinA4, or Plexin-B1 were incubated with Alkaline Phosphatase (AP)-Sema6D-Fc (Fig. 4A). AP-Sema6D-Fc specifically bound to Plexin-A1, weakly bound to Plexin-A4, but did not bind to Plexin-A2 or Plexin-B1. The results indicate that Plexin-A1 is the major receptor for Sema6D. Although Plexin-A1 forms a receptor complex with NP1 to transduce signals from Sema3A, NP1 neither bound to Sema6D nor influenced the binding of Sema6D to Plexin-A1.
Plexin-A1 expression was investigated by in situ hybridization (Fig. 4B). Plexin-A1 expression in the mouse embryo became observable at E10.5 (Fig. 4B, panel a). Cross-sections indicated Plexin-A1 expression in the lateral side of the neural fold (Fig. 4B, arrowhead in panel b), the endocardial cushion region (Fig. 4B, arrow in panel c), and the subepicardial side of the ventricular wall (Fig. 4B, arrowhead in panel c). Intense expression of Plexin-A1 in endocardial cells was observed within the cardiac cushion (Fig. 4B, arrow in panel d) and ventricular wall (Fig. 4B, arrows in panel e). Plexin-A1 expression in myocardial cells was more prominent on the epicardial side of myocardium (compact zone; Fig. $4 \mathrm{~B}$, arrowhead in panel e) than in trabeculae. Thus, although both Sema6D and Plexin-A1 are expressed in developing heart, there are some differences in their expression patterns, that is, the expression of Plexin-A1 is higher than that of Sema6D in the endocardial cells and lower in trabeculae. Consistent with the pattern of mouse Plexin-A1 expression, the chicken homolog of Plexin-A1, cPlexin-A1, was expressed in the lateral side of the neural fold (Fig. 4B, arrow in panel g) and in the cardiac tube wall within chick embryos (Fig. 4B, arrow in panel h). 
Toyofuku et al.

Figure 4. Plexin-Al binds to Sema6D and its mRNA is expressed in the developing neural and cardiac tubes and mediates the effect of Sema6D on chick embryo. (A) Micrographs of binding assays that test the binding of truncated Sema6D on HEK293 cells transfected with Plexin-A1, PlexinA2, Plexin-A4, Plexin-B1, or NP1. An alkaline phosphatase fusion with the truncated Sema6DFc domain was incubated with the transfectants; the final detection of binding was performed using alkaline phosphatase substrates. Significant staining is observed in Plexin-Al-expressing cells and cells coexpressing Plexin-A1 and NP1. Bar, $50 \mu \mathrm{m}$. (B) Expression of Plexin-A1 mRNA in E10.5 mouse embryo. (Panel a) Lateral view of E10.5 mouse embryo. Bar, $100 \mu \mathrm{m}$. (Panels $b, c)$ Crosssections through the mouse embryo shown in panel $a$. Plexin-A1 mRNA is expressed in the lateral side of the neural tube (panel $b$, arrowhead), the atrioventricular cushion (panel $c$, arrow), and the myocardial layer of cardiac ventricular wall (panel $c$, arrowhead). Bar, $100 \mu \mathrm{m}$. (Panels $d, e$ ) The magnified views of the cardiac tube. Plexin-A1 mRNA is expressed in the endocardial cells of the cardiac cushion (panel $d$, arrow) and the ventricular wall (panel $e$, arrows). The intense expression of Plexin-A1 mRNA is observed in the myocardial cells on the epicardial side of the myocardium (panel $e$, arrowhead) at higher levels than in trabeculae. Bar, $20 \mu \mathrm{m}$. (Panels $f-h$ ) Expression of cPlexin-A1 mRNA in HH stage7 (panels $f, g$ ) and HH stage 12 (panel h) chick embryo. Ventral view (panel $f$ ) and cross-sections (panels $g, h$ ) display cPlexA1 mRNA expression in the lateral side of the neural fold (panel $g$, arrow) and the cardiac ventricular wall (panel $h$, arrow). The expression pattern of cPlexin-A1 mRNA in the chick embryo is similar to that observed in mouse embryos. Bar, $100 \mu \mathrm{m}$. (C, panels $a-h) \mathrm{HH}$ stage 12 chick embryos treated with in the combination of PlexAl-myc (panels $a, c-f, h)$ and soluble Sema6D-Fc (panels $b, c, g, h)$. HH stage 4 chick embryos were treated with various combinations of soluble Sema6D-Fc $(100 \mathrm{ng} / \mathrm{mL})$ and PlexA1-myc $(10 \mu \mathrm{g} / \mathrm{mL})$. Embryos were then immunostained with anti-vMHC antibody (panels $a-c$ ) or in situ hybridized with riboprobes for VMHC1 (panel $d$ ) or AMHC1 (panel e). The neural folds of cross-sectioned embryos (panels $f-h$ ) were visualized with hemotoxylene and eosin staining. PlexAl-myc alone induces a narrowing of the cardiac cavity instead of the normal ballooning (panel $a$, arrowhead). Note that cPlexA1-myc distorted exclusively the ventricular portion (panel $d$, arrowhead) but not the atrial portion (panel $e$ ). In the presence of PlexAl-myc, embryos treated with Sema6D-Fc display neural tube closure (panel $h$ ) and an almost normal cardiac cavity (panel $c)$. Bar, $100 \mu \mathrm{m} .(D$, panels $a-e)$ HH stage 12 chick embryos treated with control (panel $a$ ) or cPlexin-A1 specific siRNA (panels $b-e$ ) in the presence of soluble Sema6D-Fc (panel c). HH stage 6 chick embryos were electroporated with either control (panel $a$ ) or cPlexin-A1 siRNAs $(40$ pmole/ $\mu \mathrm{L}$; panels $b-e)$, then incubated with soluble Sema6D-Fc (100 ng/mL; panel $c)$. Embryos were cultured until HH stage 12 . Embryos were then immunostained with anti-vMHC antibody (panels $a-c$ ) or in situ hybridized with riboprobes for VMHC1 (panel $d$ ) or AMHC1 (panel e). Embryos treated with siRNA specific for cPlexin-Al display poorly looped cardiac tube (panel $b$, arrowhead). Note that siRNA specific for cPlexin-A1 distorted exclusively the ventricular portion (panel $d$, arrowhead) but not the atrial portion (panel $e$ ). This phenotype is similar to embryos treated with siRNA specific for cSema6D (Fig. 2M,Q,R). In the presence of Sema6D-Fc, nearly normal cardiac tube formation is restored in embryos treated with siRNA specific for cPlexin-A1 (panel c). Bar, $100 \mu \mathrm{m}$.

\section{The effect of Sema6D is blocked by truncated Plexin-A1 and RNAi against Plexin-A1}

Chick embryos were cultured in the presence of soluble Sema6D alone or in combination with excess PlexA1myc or PlexA2-Flag, recombinant proteins in which the transmembrane and cytoplasmic regions of Plexin-A1 and Plexin-A2 have been replaced with a myc tag and a Flag tag, respectively (Fig. 4C; Supplementary Table 1).
When incubated with PlexA1-myc alone, embryos exhibited significantly retarded bending of the ventricular portion of cardiac tube (Fig. 4C, arrowhead in panels a,d; Supplementary Table 1), whereas PlexA2-Flag did not show any effect on the cardiac tube bending. This result indicated that signals mediated by the endogenous Plexin-A1 are critical in normal cardiac morphogenesis. PlexA1-myc substantially blocked the Sema6D-induced changes in neural and cardiac tube development (Fig. 4C, 
panels c,h). We then examined the effect of RNAi against cPlexin-A1 on Sema6D induced change of cardiac tube formation (Fig. 4D). Treatment with siRNA specific for cPlexin-A1 significantly reduced the levels of cPlexin-A1 mRNA without changing any other mRNA tested (Supplementary Fig. 2B). RNAi against cPlexin-A1 inhibited the normal ballooning and extension of the ventricular portion, leading, instead, to a short, narrow ventricular portion insufficient for normal right-side bending. (Fig. 4D, arrowheads in panels b, d; Supplementary Table 1). This phenotype is similar to that induced by RNAi against cSema6D (Fig. 2M). Furthermore, RNAimediated down-regulation of cPlexin-Al expression could prevent cardiac expansion induced by exogenous Sema6D (Fig. 4D, panel c). These results indicate that Plexin-A1 functions as a receptor for Sema6D in the cardiac tube.

We next examined the antagonism of truncated Plexin-A1 on Sema6D signals to cardiac explants (Fig. 5). Coincubation with PlexA1-myc blocked both the enhancing and suppressing effects of Sema6D on the out- growth of explants from the CT segment and the ventricle, respectively. (Fig. 5A, panels c,g). Furthermore, Sema6D did not exert an effect on the outgrowth of explants transfected with glycosylphosphatidylinositol (GPI)-anchored-Plexin-A1 (PlexA1-DN; Fig. 5A, panels d,h). RNAi against cPlexin-A1 also blocked Sema6D-induced changes in outgrowth from both cardiac explants (Fig. 5B, panels b,d). Thus, Plexin-A1 is responsible for Sema6D signal transduction in the endothelial outgrowth derived from cardiac explants.

\section{OTK and VEGFR2 are involved in Sema6D signals in the ventricle and CT segments, respectively, by forming the receptor complexes with Plexin-A1}

Plexin-A1 may form receptor complexes specific for Sema6D containing distinct components to exert opposite effects on different regions of the cardiac tube. The Drosophila Plexin-A receptor associates with OTK, a receptor tyrosine kinase-like transmembrane protein. This complex transduces Semala signals (Winberg et al.
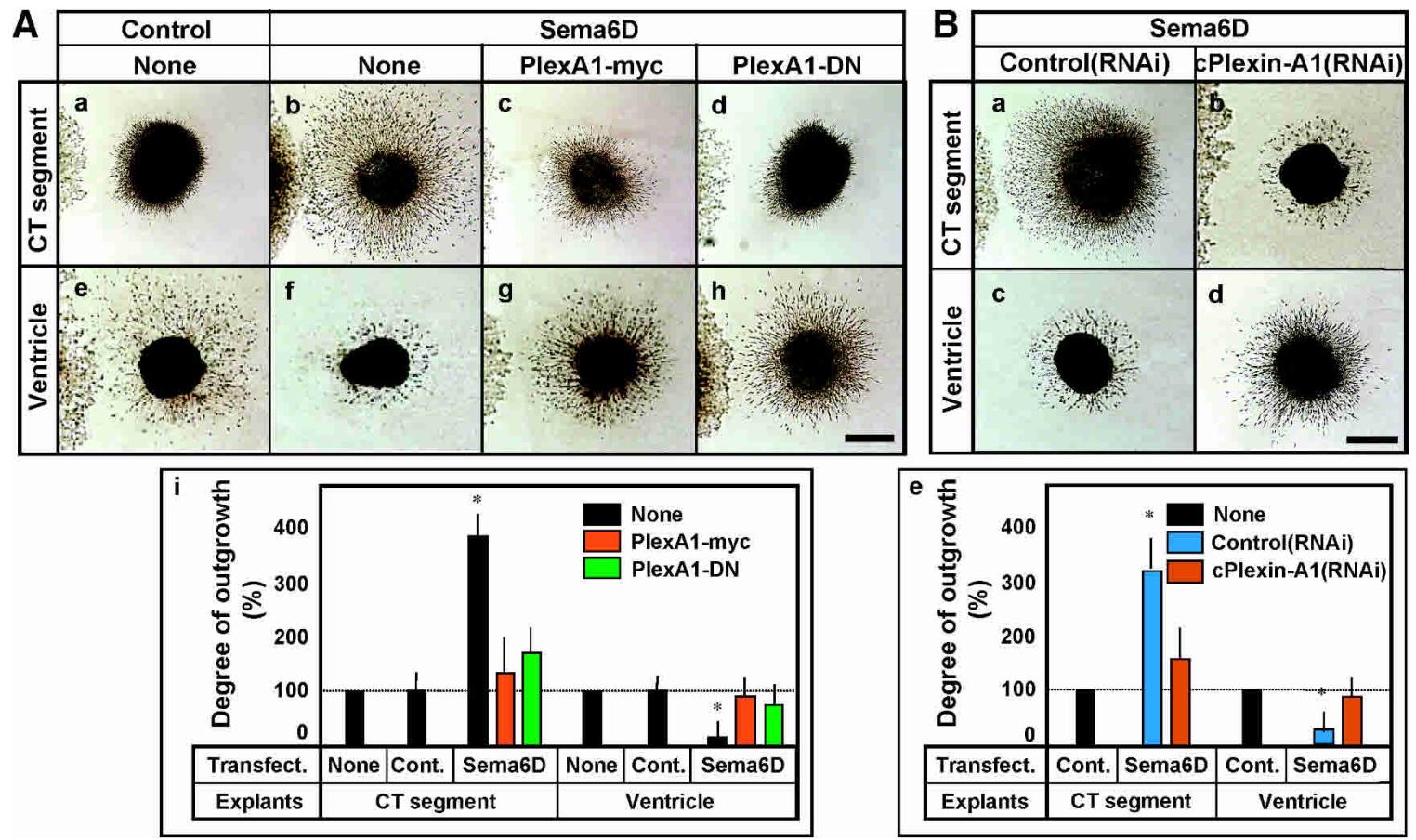

Figure 5. Truncated Plexin-A1 and RNAi against cPlexin-A1 block the effect of Sema6D on cardiac explants. $(A$, panels $a-i)$ Collagen gel coculture of cardiac explants with control (panels $a, e$ ) or Sema6D-expressing cells (panels $b-d, f-h$ ). Cardiac explants were positioned on the right, whereas cell aggregates (transfect.) were positioned on the left. Cardiac explants were visualized by phase contrast microscopy. Some cultures (panels $c, g$ ) contained PlexAl-myc, whereas in other cultures (panels $d, h$ ), explants were infected with retroviruses encoding the PlexA1-DN and placed on collagen gels. Incubation with PlexA1-myc or infection with PlexA1-DN block Sema6D-induced changes in outgrowth from both of CT segments and ventricle explants. Bar, $100 \mu \mathrm{m}$. (Panel i) Quantification of the relative degree of outgrowth from cardiac explants as a percentage of control value. Data are means \pm S.E.M. $\left({ }^{\star}\right) p<0.05$, vs. explants with control cells without reagents. $(B$, panels $a-e)$ Collagen gel cocultures of cardiac explants treated with control (panels $a, c)$ or cPlexin-A1-specific (panels $b, d$ ) siRNA with Sema6D-expressing cells (panels $a-d$ ). Cardiac tissue explants were transfected with either control or cPlexin-A1 siRNA and then placed on the collagen gels. Explants were positioned on the right, whereas cell aggregates were placed on the left. Cardiac explants were visualized by phase contrast microscopy. (Panels $b, d$ ) RNAi against cPlexin-A1 blocks Sema6D-induced changes in outgrowth from both CT segments and ventricle explants. Bar, $100 \mu$ m. (Panel e) Quantification of the relative degree of outgrowth from cardiac explants as a percentage of control value. Data are means \pm S.E.M. $\left({ }^{\star}\right) p<0.05$, vs. explants with control cells without reagents. 
Toyofuku et al.

2001). We therefore analyzed the expression of various receptor kinases and NP1 in outgrowing cells from cardiac explants (Fig. 6A). Among analyzed molecules, four molecules exhibited unique expression patterns. OTK and NP1 mRNAs were expressed predominantly in the outgrowing cells from the ventricle. In contrast, VEGFR1 and VEGFR2 mRNAs were strongly expressed in cells of the CT segment. NP1 is known to form the functional Sema3A receptor complex with Plexin-A1. However, in spite of its abundant expression, we could not detect the interaction of NP1 with Plexin-A1 in cells from the ventricle (Fig. 6B), which is in good agreement with the fact that Sema3A did not show any effect on the ventricle explants. As Drosophila OTK has been shown to interact with not only invertebrate Plexin-A, but also vertebrate Plexin-A3 and Plexin-B3, we analyzed the ability of OTK to interact with Plexin-A1. As shown in Figure 6C, an association between OTK and Plexin-A1 could be demonstrated when these molecules were transfected into HEK293 cells. We next examined the functional involvement of vertebrate OTK in Sema6D signaling by knocking down OTK expression by RNAi. Treatment with siRNA specific for chicken OTK (cOTK) significantly reduced the levels of cOTK mRNA without changing any other mRNA tested (data not shown). RNAi against cOTK blocked Sema6D-induced inhibi-
A

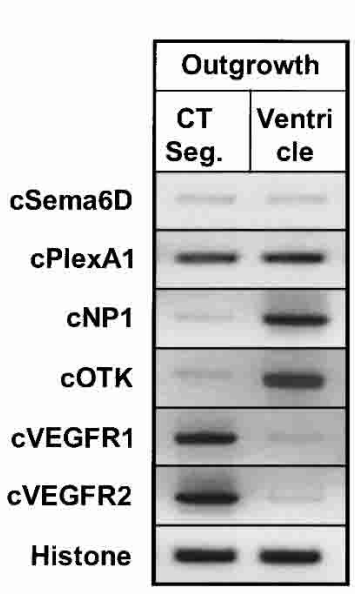

C

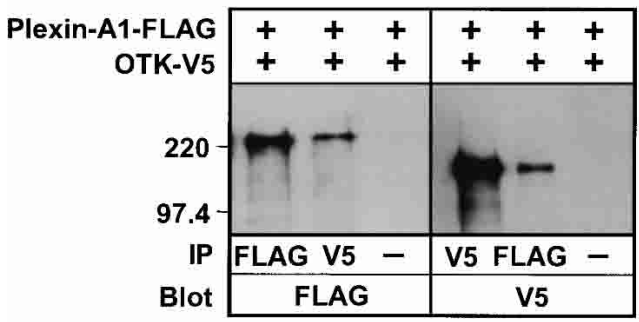

B

D
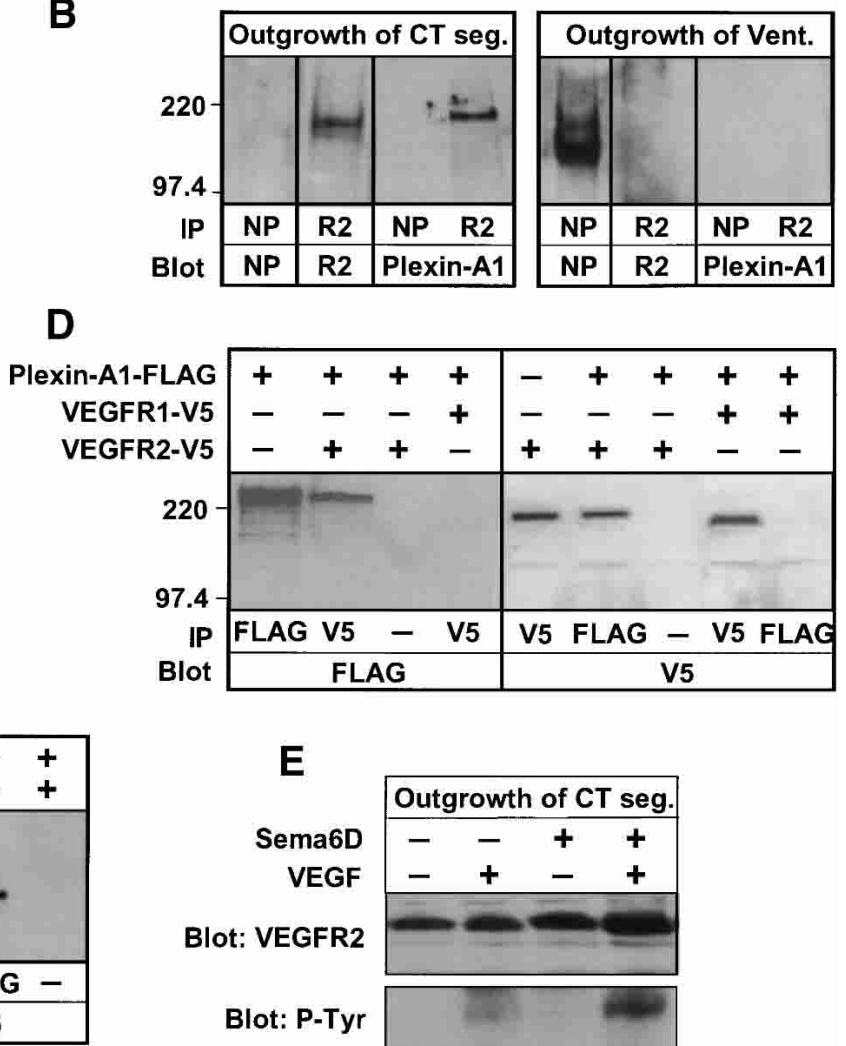

Figure 6. Plexin-A1 interacts with OTK and VEGFR2. (A) RT-PCR analysis of mRNA extracted from outgrowing cells from cardiac explants. Cardiac tissues were removed from chick embryos, placed on the collagen gel, and cultured with medium for $2 \mathrm{~d}$. After removing the core region of the explant, outgrowing cells were subjected to mRNA extraction. The outgrowing cells from ventricle explants exhibit NP1 and OTK mRNA expression, whereas cells from CT segments exhibit VEGFR1 and 2 mRNA expression. $(B)$ Association of endogenous Plexin-A1 and VEGFR2 in outgrowing cells from CT segments. Outgrowing cells were prepared as described in $A$. Lysates prepared from outgrowing cells were immunoprecipitated (IP) with anti-NP1 antibodies (NP), or anti-VEGFR2 antibodies (R2). Immunoprecipitates were blotted (Blot) with the indicated antibodies. In outgrowing cells from the CT segment, Plexin-A1 is detected in immunoprecipitates using anti-VEGFR2 antibodies, but not in immuoprecipitates using anti-NP1 antibodies in outgrowing cells from the ventricle. Molecular weight markers are indicated at left. (C) Association of Plexin-Al with OTK, following expression in HEK293 cells. Lysates prepared from transfected cells were immunoprecipitated with anti-Flag antibodies (FLAG) or anti-V5 antibodies (V5). Immunoprecipitates were blotted with the indicated antibodies. OTK is detected in lysates from cells cotransfected with Plexin-A1. Molecular weight markers are indicated at left. $(D)$ Association of Plexin-A1 with VEGFR2, following expression in HEK293 cells. Lysates prepared from transfected cells were immunoprecipitated with Flag or V5. Immunoprecipitates were blotted with the indicated antibodies. VEGFR2, but not VEGFR1, is detected in lysates from cells cotransfected with Plexin-A1. Molecular weight markers are indicated at left. (E) Tyrosine phosphorylation of VEGFR2 in outgrowing cells from CT segments. Outgrowing cells from CT segments were prepared as described in Figure 6A. Outgrowing cells were stimulated with VEGF $(50 \mathrm{ng} / \mathrm{mL})$ and/or Sema6D-Fc $(100 \mathrm{ng} / \mathrm{mL})$ for $30 \mathrm{~min}$ before harvest. Lysates of outgrowing cells were immunoprecipitated with anti-VEGFR2 antibody. Immunoprecipitates were blotted with anti-VEGFR2 antibodies and anti-phosphotyrosine antibody (p-Tyr). 
tion of migration of outgrowing cells from ventricle explants (Fig. 7A), but did not influence the effect of Sema6D on cells from CT segments (Fig. 7A), indicating that OTK is functionally coupled with Plexin-A1 in the ventricle region. We also examined the effect of RNAi against cOTK on cardiac tube formation. Treatment with siRNA specific for cOTK, which significantly reduced the expression of cOTK mRNA (Supplementary Fig. $2 \mathrm{~B} \mid$, resulted in the failure of bending and expansion of the ventricular region (Fig. 7C, arrowheads in panels $\mathrm{b}, \mathrm{d}$; Supplementary Table 1). This phenotype is similar to those of chick embryos treated with siRNA specific for cSema6D (Fig. 2M) or cPlexin-A1 (Fig. 4D, panel b). These results suggest that the morphological effect of Sema6D on the ventricular region is mediated through the Plexin-A1-OTK receptor complex.

We then examined the physical interaction of PlexinA1 with VEGFR1 or VEGFR2. VEGFR2, but not VEGFR1, physically associated with Plexin-A1 in cells transfected with these molecules (Fig. 6D). The interaction between endogenous VEGFR2 and Plexin-A1 was also demonstrated in outgrowing cells from CT explants (Fig. 6B). The addition of truncated VEGFR2 (VEGFR2-myc) and RNAi against chicken VEGFR2 [cVEGFR2(RNAi)] in car-
A

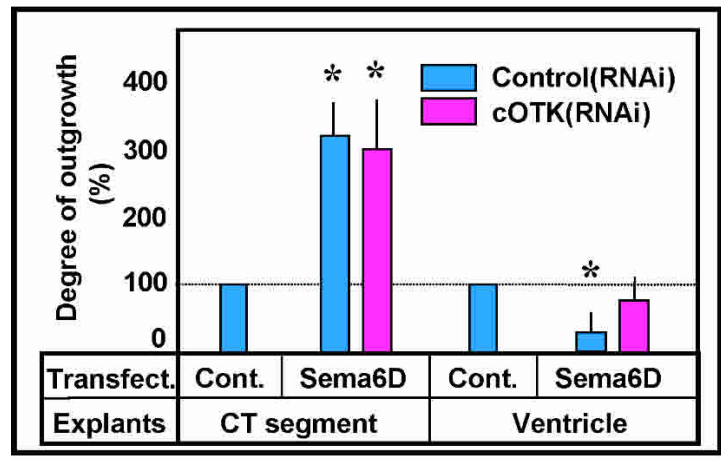

B

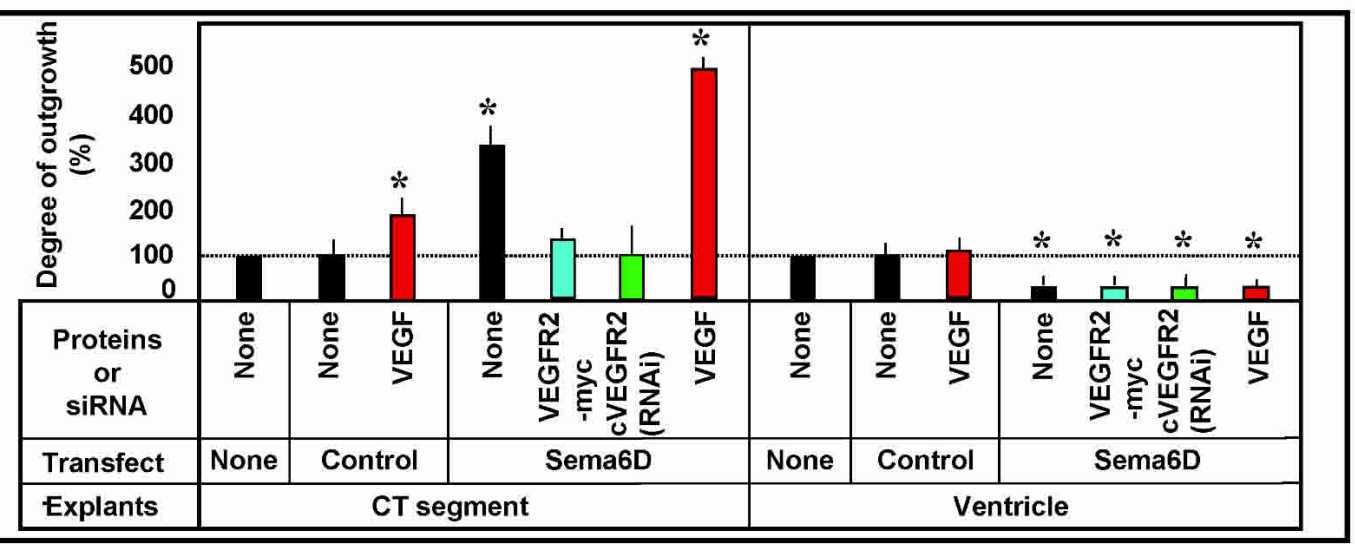

Figure 7. Differential association of Plexin-A1 with OTK and VEGFR2 conveys the opposite effects of Sema6D on ventricle and CT segment. $(A)$ Quantification of the relative degree of outgrowth from cardiac explants as a percentage of control value. Collagen gel coculture was performed using cardiac explants treated with control or cOTK-specific siRNA in the presence of control or Sema6Dexpressing cells. RNAi against cOTK blocks Sema6D-induced changes in outgrowth from ventricle explants but not from CT segments. Data are means \pm S.E.M. $\left(^{\star}\right) p<0.05$, vs. explants with control cells without reagents. $(B)$ Quantification of the relative degree of outgrowth from cardiac explants as a percentage of control value. Collagen gel coculture was performed using cardiac explants with control or Sema6D-expressing cells. Some culture medium contained VEGF ( $50 \mathrm{ng} / \mathrm{mL}$ ) or truncated VEGFR2-myc (10 $\mu \mathrm{g} / \mathrm{mL})$. In other cultures, explants were treated with siRNA specific for cVEGFR2. CT segments exhibit increased outgrowth by VEGF alone, but decrease Sema6D-induced outgrowth by incubation with truncated VEGFR2-myc or treatment with siRNA specific for VEGFR2. Sema6D-induced outgrowth is augmented by VEGF. Ventricle explants do not demonstrate significant changes in Sema6D-induced outgrowth as well as control outgrowth by the treatment of VEGF and VEGFR2 mutants. Data are means \pm S.E.M. $\left({ }^{\star}\right) p<0.05$, vs. explants with control cells without reagents. $(C)$ HH stage 12 chick embryos treated with control (panel $a$ ), cOTK (panels $b, d, e)$, or cVEGFR2 specific siRNA (panel c). HH stage 6 chick embryos were electroporated with either control (panel $a$ ), OTK siRNAs (40 pmole $/ \mu \mathrm{L}$; panels $b, d, e)$, or cVEGFR2 siRNAs $(40$ pmole $/ \mu \mathrm{L}$; panel $c)$. Embryos were cultured until HH stage 12 . Embryos were then immunostained with anti-vMHC antibody (panels $a-c$ ) or in situ hybridized with riboprobes for VMHC1 (panel $d$ ) or AMHC1 (panel $e$ ). Embryos treated with siRNA specific for OTK display poorly looped cardiac tube (panel $b$, arrowhead). Note that siRNA specific for cOTK distorted exclusively the ventricular portion (panel $d$, arrowhead) but not the atrial portion (panel $e$ ). (Panel $c$ ) In contrast, embryos treated with cVEGFR2 specific siRNA display nearly normal cardiac tube. Bar, $100 \mu \mathrm{m}$. 
diac explants abrogated the effect of Sema6D on CT segment explants, but not on ventricle explants (Fig. 7B). VEGF is produced by CT segment cells (Miquerol et al. 1999|. VEGF substantially enhanced migration of outgrowing cells from CT segment explants in the absence of Sema6D (Fig. 7B). The combination of exogenous Sema6D and VEGF enhanced outgrowth from CT segment explants more effectively than either Sema6D or VEGF alone (Fig. 7B). These results suggest that Sema6D binding to Plexin-A1 enhances the VEGFR2-signaling pathway, inducing the enhanced outgrowth of endothelial cells from CT segments only. To test this possibility, we analyzed the effect of exogenous Sema6D on the tyrosine phosphorylation of VEGFR2. As shown in Figure 6E, Sema6D synergistically enhanced VEGF-induced phosphorylation of VEGFR2, indicating that the collaboration of Sema6D and VEGF signals through the Plexin-A1-VEGFR2 complex. Finally, we examined the role of VEGFR2 in cardiac tube formation by RNAi. As shown in Figure 7C (panel c), normal bending and expansion of the cardiac tube was observed in embryos treated with siRNA specific for VEGFR2, although this treatment significantly reduced the expression of VEGFR2 mRNA (Supplementary Fig. 2B). This indicates that VEGFR2 is dispensable for Sema6D signaling during this stage of cardiac development, and rather suggests that the Plexin-A1-VEGFR2 complex may mediate the Sema6D activity at the later stage, particularly in endocardiac cushion formation, in which VEGF is known to be critically involved (Dor et al. 2001; Johnson et al. 2003).

\section{Discussion}

\section{Biological activities of Sema6D}

Sema6D has been cloned previously by Qu et al. (2002) and shown to induce the growth cone collapse of dorsal root ganglion and hyppocampal neurons, but not cortical neurons. In this study, we were able to show that Sema6D affects the embryonic cardiac morphogenesis by utilizing ex vivo culture of chick embryos. Sema6D was expressed in the developing tubular heart and in the neural fold of both mice and chicks. Both RNAi-mediated Sema6D suppression and Sema6D overexpression in chick embryos caused morphological abnormalities of the cardiac tube, indicating that a tight regulation of Sema6D expression is critical for cardiac tube formation. Moreover, we revealed that Sema6D acts in two distinct manners, stimulatory and inhibitory, upon migration of endothelial cells derived from different regions of cardiac tubes. However, it remains to be clarified whether the effects of Sema6D on migration of endothelial cells are solely responsible for the phenotypes induced by Sema6D overexpression or knockdown, as there is a possibility that Sema6D may also act on myocardial cells. In addition, the Sema6D-induced defect in neural-tube closure suggests the involvement of this molecule in neural tube formation. Sema6D, hence, is evidently involved in cardiac morphogenesis and possesses the pleiotropic functions.

\section{Plexin-A1 functions as a receptor for Sema6D}

We demonstrated in this study that Plexin-A1 interacts with Sema6D. Drosophila Plexin-A serves as a receptor for Semala (Winberg et al. 1998), a member of the Drosophila class I semaphorin subfamily. This subfamily is structurally closer to the class VI subfamily than to other vertebrate semaphorin subfamilies (Zhou et al. 1997), suggesting a close phylogenetical relationship between these two subfamilies, thereby justifying the interaction found between Sema6D and Plexin-A1. The finding also suggests the possibility of additional pairings between mammalian class VI semaphorin and Plexin-A subfamily members.

The effects of Sema6D on neural and cardiac tube formation in early embryos and on the outgrowth of cadiac explants were blocked by truncated forms of Plexin-A1. The effect of Sema6D on cardiac tube formation or on outgrowth of cardiac explants could also be blocked by treatment with RNAi against cPlexin-A1. These results suggest that Plexin-A1 mediates the effect of Sema6D on neural tube formation and cardiac morphogenesis. Furthermore, treatment of chick embryos with RNAi against cPlexin-A1 or soluble Plexin-A1 retarded the bending of cardiac tubes, indicating that endogenous Plexin-A1 is essential for normal cardiac morphogenesis.

\section{Differential association of Plexin-A1 with OTK or VEGFR2 determines the region-specific biological activities of Sema6D}

Sema6D exerted a migration-inhibitory activity upon ventricle explants, in which we detected high levels of NP1 and OTK. However, Plexin-A1 did not interact with NP1, but instead with OTK in this region, implying that the association of Plexin-A1 with OTK is necessary for the inhibitory effect of Sema6D on the outgrowing cells of the ventricle region. Thus, in this region, Plexin-A1 may form the Sema6D receptor complex with OTK, which interferes with the association of NP1. The present result is reminiscent of the previous finding that Drosophila Plexin-A-OTK complex mediates the chemorepulsive cue of Semala. Sema6D-mediated inhibition of cell migration observed in this study may share a similar molecular mechanism with chemorepulsion induced by semaphorin molecules. We observed that Sema6D induces high levels of Rac-GTPase and low levels of Rho-GTPase activities in ventricle explant cells (Supplementary Fig. 4), similar to a previous observation for Sema3A-treated nervous cells (Messersmith et al. 1995).

On the other hand, when Sema6D exerted the stimulatory effect on the migration of cells from the CT segments, we found that Plexin-A1 interacts with VEGFR2 in the CT segment. A truncated form of either Plexin-A1 or VEGFR2 blocked the effect of Sema6D on explants from the CT segment. VEGF, which plays multiple essential roles in blood-vessel formation, is expressed in the embryonic myocardial tube (Miquerol et al. 1999). Tight control of VEGF expression is crucial for normal 
cardiac morphogenesis (Carmeliet et al. 1996; Miquerol et al. 2000). Like Sema6D, VEGF also enhanced the outgrowth from the CT segments. Moreover, Sema6D synergistically enhanced VEGF-induced tyrosine phosphorylation of VEGFR2. These observations indicate that Sema6D may function as a modifier of VEGF signals through the Plexin-A1-VEGFR2 complex in the CT segment.

\section{Physiological relevance of Sema6D} on cardiac formation

During cardiac morphogenesis, the cardiac linear tube, composed of cardiac precursor cells migrated from the anterior lateral plate mesoderm, is patterned along the anterior-posterior axis into the conotruncus (outflow tract), the right and left ventricles, and the atria. The cardiac tube then differentiates into the heart through a complex process involving right bending, ballooning of the ventricular segments, and fusion of the CT and atrioventricular segments. The present study demonstrates an essential role for Sema6D in cardiac tube looping and ventricular ballooning. In addition, Sema6D exerts inhibitory and promoting effects on cell migration of ventricular and CT segments, respectively. These findings suggest that Sema6D mediates region-specific control of the migration of proliferating endothelial cells in different segments to coordinate cardiac tube looping, particularly ventricular ballooning, although we cannot exclude a possible effect of Sema6D on the myocardium.

Sema6D induced ventricular expansion, accompanied by a thinning of the myocardial layers and poor trabeculation. Maturation of the ventricular wall requires the development of myocardial layers; immature myocardial cells proliferate in the compact zone, whereas differentiated myocardial cells migrate into the lumen later (trabeculation; Sedmera et al. 2000). Our findings raise the possibility that Sema6D, expressed throughout myocardial layers, may inhibit the migration of proliferating endocardial and myocardial cells from the compact zone through the Plexin-A1-OTK complex. Further studies, however, will be required to determine the direct effect of Sema6D on myocardial cells. Alternatively, the migratory changes of endocardial cells induced by alterations in Sema6D may indirectly modify the morphology of the myocardial layer, as the endocardial layer is known to affect the formation of the myocardial layer (Brutsaert and Andries 1992).

CT and atrioventricular valve segments fuse, forming the endocardial cushion. The magnitude of this structure is precisely regulated by the balance between VEGF-mediated endocardial cell proliferation (Johnson et al. 2003) and TGF $\beta$-mediated mesenchymal transformation (Brown et al. 1999). High expression of VEGF in the myocardium coincides with regions such as the endocardial cushion in which endocardial cells proliferate (Miquerol et al. 1999). Taken together with the fact that in ovo ectopic expression starting at $\mathrm{HH}$ stage 12 resulted in substantial extension of endocardial cushion, the collaboration between Sema6D and VEGF on the CT seg- ment also suggests a possible involvement of Sema6D in endocardial cushion formation.

Thus, our findings present clear evidence that the semaphorin system is critical in cardiac morphogenesis by regulating cell migration. Furthermore, the finding that the region-specific association of its canonical receptor with various receptor-type tyrosine kinases determines pleiotropic activities of Sema6D provides a new insight into the molecular basis of semaphorin signals.

\section{Materials and methods}

\section{Construction of cDNAs}

To isolate mouse cDNAs encoding semaphorins and plexins from total mouse heart cDNAs, we used an RT-PCR method using degenerative oligonucleotide primers for semaphorins as described previously (Puschel et al. 1995) and 5'-AACAG(AG) TC(AG)TC(CG)AC(AG)AA(CT)TT(CT)TG-3' and 5'-GTGAA (AG)GT(ACG)CT(AG)GA(CT)TGTGA/CT)AC-3' for plexins. Use of a clone homologous to a class VI semaphorin as a probe identified full ORF clones by hybridization. To isolate the chicken homolog of Sema6D (cSema6D) and Plexin-A1 (cPlexin-A1), chicken EST sequences were used as probes for hybridization of a chicken embryo cDNA library.

\section{Construction of transfectants}

To generate Sema6D-expressing transfectants, full-length Sema6D or cSema6D cDNA fragments lacking stop codons were ligated into the pcDNA3.1/myc-HisA or pcDNA3.1/V5HisA expression vectors (Invitrogen), and resulting plasmids were transfected into HEK293 cells by Fugene-6 (Roche Molecular Biochemicals). Stable cell lines overexpressing the proteins encoded by these plasmids were established by selection with $800 \mu \mathrm{g} / \mathrm{mL}$ G418 (Sigma). To construct a PlexA1-DN, the sequences encoding the ectodomain of the molecule were synthesized by PCR and ligated to myc and GPI coding sequences. These PCR fragments were ligated into the RCAS retroviral vector (the kind gift of Dr. Stephen H. Hughes, National Cancer Institute, Frederick, MD). The cardiac explants dissected from chick embryos were transfected with retrovirus with Fugene-6 reagent.

\section{Generation and production of soluble recombinant proteins}

To produce soluble Fc-fused proteins, the extracellular domain of the membrane type protein were ligated into the pEFBos human IgG1 Fc cassette (Suda and Nagata 1994). These constructs were transfected into P3U1 plasmacytoma cells by electroporation. Fc-fused proteins were purified from culture supernatants using protein-A Sepharose (Pharmacia). To produce soluble myc-tagged proteins, PCR fragments were ligated into the pSecTag2 vector (Invitrogen) and transfected into HEK293 cells. Myc-tagged proteins were purified from culture supernatants using a His-binding column.

\section{Chicken embryo cultures and collagen gel culture assay}

Fertilized chicken eggs (Takeuchi Farm Inc.) were incubated at $38^{\circ} \mathrm{C}$ in high humidity. Embryos, staged according to the Hamburger and Hamilton method (Hamburger and Hamilton 1951), were removed from eggs and placed ventral side up in culture dishes. To study the loss of semaphorin function on early cardiogenesis, embryos in culture dishes were treated with siRNA 
by electroporation. In some experiments, embryos were implanted with aggregates of transfected cells $\left(10^{6}\right.$ cells/aggregate $)$ or incubated with recombinant proteins $(100 \mathrm{ng} / \mathrm{mL})$ and allowed to develop in a humidified incubator. To study the effects of semaphorins on the later stages of embryogenesis, following the windowing of eggs, transfectants $\left(10^{6}\right.$ cells $)$ were injected into the amniotic cavity.

Collagen was prepared as described previously (Bernanke and Markwald 1982). The CT segment and the ventricle of the heart from $\mathrm{HH}$ stage 14 chick embryos were dissected and placed onto collagen gels next to the transfectants. After polymerization, cells were overlaid with a drop of collagen.

\section{In vitro $R N A i$}

siRNAs specific for control, cSema6D, cPlexin-A1, cVEGFR2, and cOTK were chemically synthesized, then electroporated into chick embryos using electroporator (CUY-21, BEX Co.; Elbashir et al. 2001; Pekarik et al. 2003). Tungsten needles, serving as an anode, and cathode electrodes were placed on either side of the primitive cardiac tube of $\mathrm{HH}$ stage 7 chick embryos. As the siRNA solution ( $40 \mathrm{pmole} / \mu \mathrm{L})$ was being injected into the cardiac tube with a sharp glass pipette, electric pulses were applied (8 V, $90 \mathrm{msec}$, three times). To introduce siRNAs into cardiac explants; cardiac explants dissected from $\mathrm{HH}$ stage 9 chick embryos were incubated in a mixture of RNA and OligofectAMINE Reagent (Invitrogen). After incubation for $16 \mathrm{~h}$, cell aggregates expressing Sema6D were placed proximal to the transfected explants.

\section{Antibodies, immunoprecipitation, and immunohistochemistry}

We utilized the following commercially available antibodies: mouse monoclonal anti-vMHC antibody (Chemicon International, Inc.), rabbit polyclonal anti-factorVIII antibody (DAKO), rabbit polyclonal anti-fibrillin-2 antibody (EPL), a rabbit polyclonal antibody raised against the Ser10 phosphopeptide of histone H3 (Upstate Biotechnology), rabbit polyclonal anti-Flk-1 (VEGFR2) antibody (Santa-Cruz Inc.), goat polyclonal antineuropilin antibody (Santa-Cruz Inc.), goat polyclonal antiPlexin-A1 antibody (Santa-Cruz Inc.), and mouse monoclonal anti-phosphotyrosine antibody (Santa-Cruz Inc.). Immunoprecipitation was performed according to standard protocols. Whole-mount immunohistochemistry was performed as described previously (Sharpe and Mason 1998).

\section{In situ hybridization}

Chick and mouse embryos at the desired stages were fixed and then hybridized with DIG-labeled antisense RNA probes as described previously (Nieto et al. 1996). The cDNA sequences of cSema6D, cPlexin-A1, VMHC1, AMHC1, Sema6D, and PlexinAl were used to transcribe cRNAs for in situ hybridization probes.

\section{Semaphorin-alkaline phosphatase-binding assay}

Semaphorin-alkaline phosphatase-binding assay was performed as described previously (Tamagnone et al. 1999). The extracellular domain of the Sema6D was expressed as a fusion protein with placental-secreted alkaline phosphatase at the $\mathrm{N}$ terminus and Fc-domain at the $\mathrm{C}$ terminus (AP-Sema6D-Fc). The APSema6D-Fc protein was purified from culture supernatants using protein-A Sepharose. To assess binding, HEK293 cells were transiently transfected with expression vectors encoding Plex- ins and NP1. After incubation with AP-Sema6D-Fc, cells were fixed and incubated at $65^{\circ} \mathrm{C}$ for $100 \mathrm{~min}$ to inactivate endogenous AP activity. Cells expressing Plexin, which bound to APSema6D-Fc, were detected histologically.

\section{Image analysis and statistics}

To measure the area of explant outgrowth, we used NIHIMAGE software to draw a region of interest (ROI) around the outgrowth and explant core; the area contained within the ROI was then quantitated. The area of the explant core was subtracted from the total area to produce the total outgrowth area. To quantify the degree of outgrowth, the field surrounding each explant was divided into four quadrants. The amount of outgrowth in the quadrants proximal to the transfectant aggregates was then measured. The data are expressed as means \pm S.E.M. from a minimum of five independent experiments. Student's $t$-test was used for data comparison, with a significant level of $p<0.05$.

\section{Acknowledgments}

We thank Ms. K. Kubota for excellent secretarial assistance. We also thank S.H. Hughes for RCAS retroviral vector, S. Nagata for pEFBos human IgG1 Fc cassette clone, M. Uchikawa, J. Takeuchi, and T. Ogura for advice on the electroporation technique, K. Yashiro and $\mathrm{H}$. Hamada for advice on in situ hybridization technique, and $M$. Hibi for critical reading of this manuscript. This study was supported by research grants from the Ministry of Education, Science, and Culture, Japan and from CREST to T.T., A.K., and H.K.

The publication costs of this article were defrayed in part by payment of page charges. This article must therefore be hereby marked "advertisement" in accordance with 18 USC section 1734 solely to indicate this fact.

\section{References}

Behar, O., Golden, J.A., Mashimo, H., Schoen, F.J., and Fishman, M.C. 1996. Semaphorin III is needed for normal patterning and growth of nerves, bones and heart. Nature 383: 525-528.

Bernanke, D.H. and Markwald, R.R. 1982. Migratory behavior of cardiac cushion tissue cells in a collagen-lattice culture system. Dev. Biol. 91: 235-245.

Brown, C.B., Boyer, A.S., Runyan, R.B., and Barnett, J.V. 1999. Requirement of type III TGF- $\beta$ receptor for endocardial cell transformation in the heart. Science 283: 2080-2082.

Brutsaert, D.L. and Andries, L.J. 1992. The endocardial endothelium. Am. J. Physiol. 263: H985-H1002.

Carmeliet, P., Ferreira, V., Breier, G., Pollefeyt, S., Kieckens, L., Gertsenstein, M., Fahrig, M., Vandenhoeck, A., Harpal, K., Eberhardt, C., et al. 1996. Abnormal blood vessel development and lethality in embryos lacking a single VEGF allele. Nature 380: 435-439.

Castellani, V., De Angelis, E., Kenwrick, S., and Rougon, G. 2002. Cis and trans interactions of L1 with neuropilin-1 control axonal responses to semaphorin 3A. EMBO J. 21: 63486357.

Christensen, C.R., Klingelhofer, J., Tarabykina, S., Hulgaard, E.F., Kramerov, D., and Lukanidin, E. 1998. Transcription of a novel mouse semaphorin gene, $\mathrm{M}$-semaH, correlates with the metastatic ability of mouse tumor cell lines. Cancer Res. 58: $1238-1244$.

Comeau, M.R., Johnson, R., DuBose, R.F., Petersen, M., Gearing, P., VandenBos, T., Park, L., Farrah, T., Buller, R.M., Co- 
hen, J.I., et al. 1998. A poxvirus-encoded semaphorin induces cytokine production from monocytes and binds to a novel cellular semaphorin receptor, VESPR. Immunity 8: 473-482.

Dor, Y., Camenisch, T.D., Itin, A., Fishman, G.I., McDonald, J.A., Carmeliet, P., and Keshet, E. 2001. A novel role for VEGF in endocardial cushion formation and its potential contribution to congenital heart defects. Development 128: $1531-1538$.

Elbashir, S.M., Martinez, J., Patkaniowska, A., Lendeckel, W., and Tuschl, T. 2001. Functional anatomy of siRNAs for mediating efficient RNAi in Drosophila melanogaster embryo lysate. EMBO J. 20: 6877-6888.

Giordano, S., Corso, S., Conrotto, P., Artigiani, S., Gilestro, G., Barberis, D., Tamagnone, L., and Comoglio, P.M. 2002. The semaphorin $4 \mathrm{D}$ receptor controls invasive growth by coupling with Met. Nat. Cell. Biol. 4: 720-724.

Hamburger, V. and Hamilton, H.L. 1951. A series of normal stages in the development of the chick embryo. J. Morph. 88: 49-92.

Johnson, E.N., Lee, Y.M., Sander, T.L., Rabkin, E., Schoen, F.J., Kaushal, S., and Bischoff, J. 2003. NFATc1 mediates vascular endothelial growth factor-induced proliferation of human pulmonary valve endothelial cells. J. Biol. Chem. 278: 16861692.

Kagoshima, M. and Ito, T. 2001. Diverse gene expression and function of semaphorins in developing lung: Positive and negative regulatory roles of semaphorins in lung branching morphogenesis. Genes Cells 6: 559-571.

Kolodkin, A.L., Matthes, D.J., and Goodman, C.S. 1993. The semaphorin genes encode a family of transmembrane and secreted growth cone guidance molecules. Cell 75: 13891399.

Kumanogoh, A., Watanabe, C., Lee, I., Wang, X., Shi, W., Araki, H., Hirata, H., Iwahori, K., Uchida, J., Yasui, T., et al. 2000. Identification of CD72 as a lymphocyte receptor for the class IV semaphorin CD100: A novel mechanism for regulating B cell signaling. Immunity 13: 621-631.

Kumanogoh, A., Marukawa, S., Suzuki, K., Takegahara, N., Watanabe, C., E. Ch'ng, E., Ishida, I., Fujimura, H., Sakoda, S., Yoshida, K., et al. 2002. Class IV semaphorin Sema4A enhances T-cell activation and interacts with Tim-2. Nature 419: 629-633.

Messersmith, E.K., Leonardo, E.D., Shatz, C.J., Tessier-Lavigne, M., Goodman, C.S., and Kolodkin, A.L. 1995. Semaphorin III can function as a selective chemorepellent to pattern sensory projections in the spinal cord. Neuron 14: 949-959.

Miao, H.Q., Soker, S., Feiner, L., Alonso, J.L., Raper, J.A., and Klagsbrun, M. 1999. Neuropilin-1 mediates collapsin-1/ semaphorin III inhibition of endothelial cell motility: Functional competition of collapsin-1 and vascular endothelial growth factor-165. J. Cell. Biol. 146: 233-242.

Miquerol, L., Gertsenstein, M., Harpal, K., Rossant, J., and Nagy, A. 1999. Multiple developmental roles of VEGF suggested by a LacZ-tagged allele. Dev. Biol. 212: 307-322.

Miquerol, L., Langille, B.L., and Nagy, A. 2000. Embryonic development is disrupted by modest increases in vascular endothelial growth factor gene expression. Development 127: 3941-3946.

Nieto, M.A., Patel, K., and Wilkinson, D.G. 1996. In situ hybridization analysis of chick embryos in whole mount and tissue sections. Methods Cell. Biol. 51: 219-235.

Pasterkamp, R.J. and Kolodkin, A.L. 2003. Semaphorin junction: Making tracks toward neural connectivity. Curr. Opin. Neurobiol. 13: 79-89.

Pekarik, V., Bourikas, D., Miglino, N., Joset, P., Preiswerk, S., and Stoeckli, E.T. 2003. Screening for gene function in chicken embryo using RNAi and electroporation. Nat. Biotechnol. 21: 93-96.

Puschel, A.W., Adams, R.H., and Betz, H. 1995. Murine semaphorin $\mathrm{D} /$ collapsin is a member of a diverse gene family and creates domains inhibitory for axonal extension. Neuron 14: 941-948.

Qu, X., Wei, H., Zhai, Y., Que, H., Chen, Q., Tang, F., Wu, Y., Xing, G., Zhu, Y., Liu, S., et al. 2002. Identification, characterization, and functional study of the two novel human members of the semaphorin gene family. J. Biol. Chem. 277: 35574-35585.

Schultheiss, T.M. and Lassar, A.B. 1999. Vertebrate heart induction. In Heart development (eds. R.P. Harvey and N. Rosenthal), pp. 51-62. Academic Press, San Diego, CA.

Sedmera, D., Pexieder, T., Vuillemin, M., Thompson, R.P., and Anderson, R.H. 2000. Developmental patterning of the myocardium. Anat. Rec. 258: 319-337.

Semaphorin Nomenclature Committee. 1999. Unified nomenclature for the semaphorins/collapsins. Cell 97: 551-552.

Sharpe, P.T. and Mason, I. 1998. Immunohistochemistry on whole embryo. In Molecular embryology: Methods and protocols (ed. I. Mason), pp. 663-666. Humana Press, Inc., Tottawa, NJ.

Shi, W., Kumanogoh, A., Watanabe, C., Uchida, J., Wang, X., Yasui, T., Yukawa, K., Ikawa, M., Okabe, M., Parnes, J.R., et al. 2000. The class IV semaphorin CD100 plays nonredundant roles in the immune system: Defective $\mathrm{B}$ and $\mathrm{T}$ cell activation in CD100-deficient mice. Immunity 13: 633-642.

Suda, T. and Nagata, S. 1994. Purification and characterization of the Fas-ligand that induces apoptosis. I. Exp. Med. 179: $873-879$.

Takahashi, T., Fournier, A., Nakamura, F., Wang, L.H., Murakami, Y., Kalb, R.G., Fujisawa, H., and Strittmatter, S.M. 1999. Plexin-neuropilin-1 complexes form functional semaphorin-3A receptors. Cell 99: 59-69.

Tamagnone, L., Artigiani, S., Chen, H., He, Z., Ming, G.I., Song, H., Chedotal, A., Winberg, M.L., Goodman, C.S., Poo, M., et al. 1999. Plexins are a large family of receptors for transmembrane, secreted, and GPI-anchored semaphorins in vertebrates. Cell 99: 71-80.

Tse, C., Xiang, R.H., Bracht, T., and Naylor, S.L. 2002. Human Semaphorin 3B (SEMA3B) located at chromosome 3p21.3 suppresses tumor formation in an adenocarcinoma cell line. Cancer Res. 62: 542-546.

Winberg, M.L., Noordermeer, J.N., Tamagnone, L., Comoglio, P.M., Spriggs, M.K., Tessier-Lavigne, M., and Goodman, C.S. 1998. Plexin A is a neuronal semaphorin receptor that controls axon guidance. Cell 95: 903-916.

Winberg, M.L., Tamagnone, L., Bai, J., Comoglio, P.M., Montell, D., and Goodman, C.S. 2001. The transmembrane protein Off-track associates with Plexins and functions downstream of Semaphorin signaling during axon guidance. Neuron 32: 53-62.

Zhou, L., White, F.A., Lentz, S.I., Wright, D.E., Fisher, D.A., and Snider, W.D. 1997. Cloning and expression of a novel murine semaphorin with structural similarity to insect semaphorin I. Mol. Cell. Neurosci. 9: 26-41. 


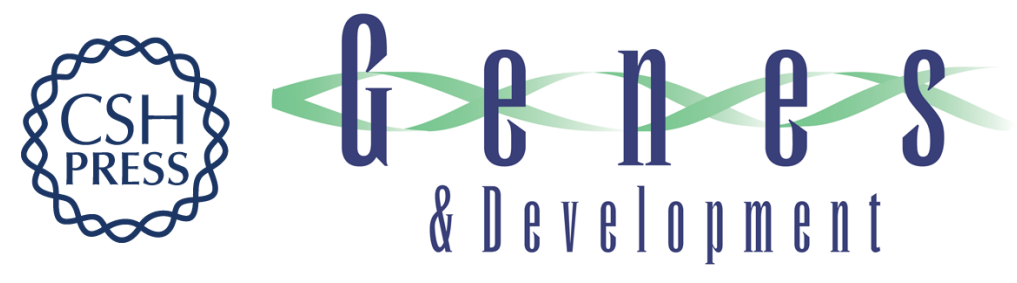

\section{Dual roles of Sema6D in cardiac morphogenesis through region-specific association of its receptor, Plexin-A1, with off-track and vascular endothelial growth factor receptor type 2}

Toshihiko Toyofuku, Hong Zhang, Atsushi Kumanogoh, et al.

Genes Dev. 2004, 18:

Access the most recent version at doi:10.1101/gad.1167304

Supplemental Material

References License

Email Alerting Service
http://genesdev.cshlp.org/content/suppl/2004/02/20/1167304.DC1

This article cites 35 articles, 11 of which can be accessed free at: http://genesdev.cshlp.org/content/18/4/435.full.html\#ref-list-1

Receive free email alerts when new articles cite this article - sign up in the box at the top right corner of the article or click here.

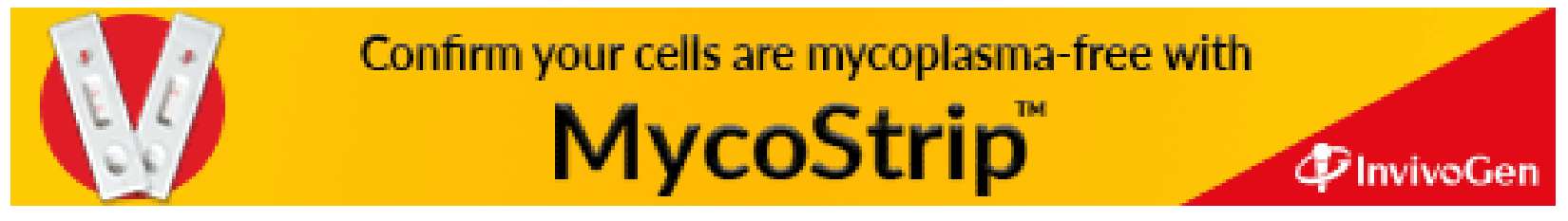

